\title{
Effektive Reanimation durch richtige Kommunikation
}

\section{Anna Henn}

Bei einer innerklinischen Reanimation treffen normalerweise Teammitglieder aus unterschiedlichen Abteilungen aufeinander, die in dieser Zusammensetzung noch nie miteinander gearbeitet haben. Diese Tatsache und die Stresssituation, in der sich alle Beteiligten befinden, können die Kommunikation erheblich erschweren, was fatale Folgen für den Patienten haben kann. Eine bestimmte Kommunikationsstrategie und der Einsatz eines Teamleiters können zur Verbesserung der Reanimationsqualität beitragen.

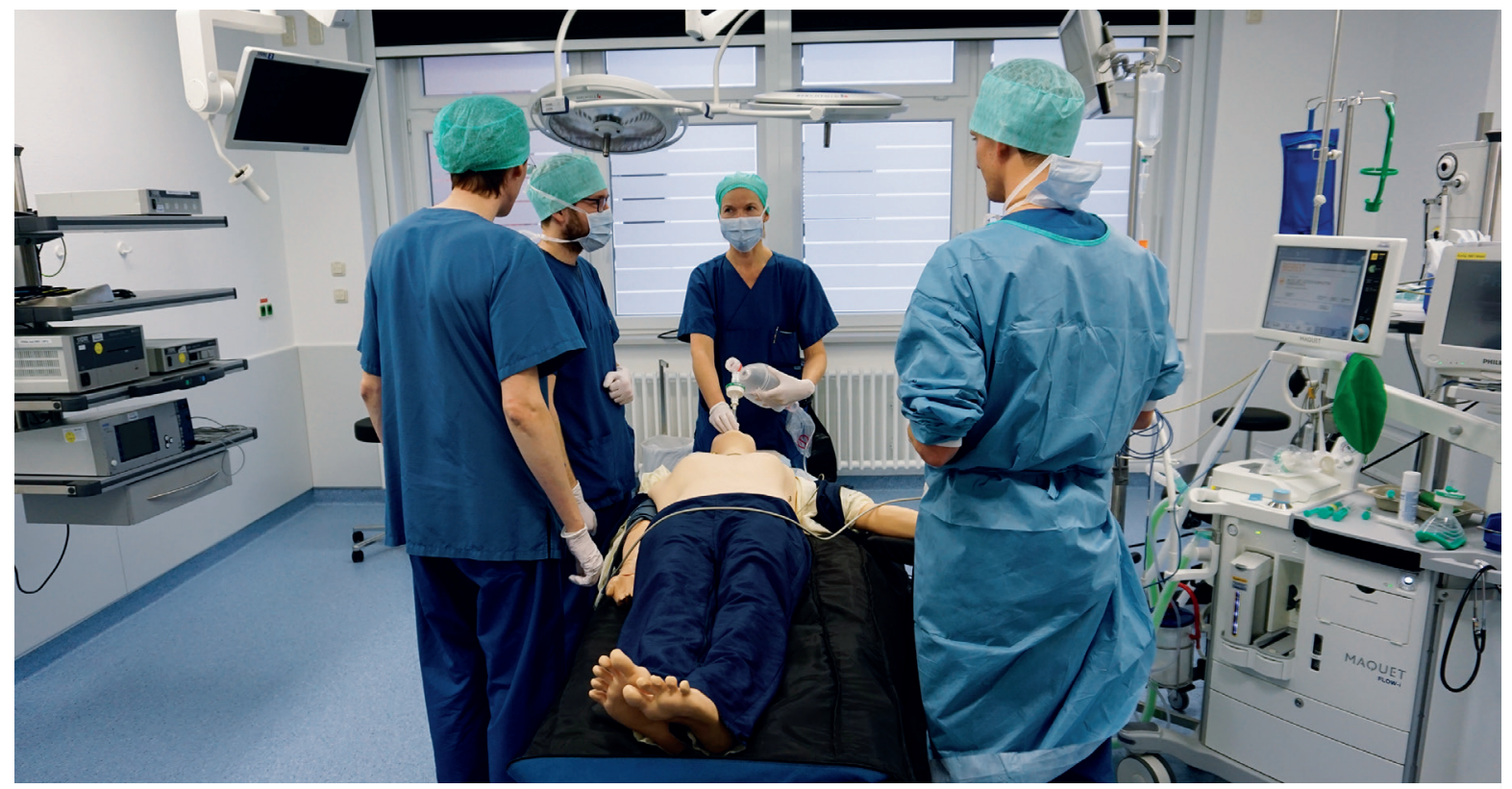

Beim Simulationstraining im OP übt das Team die Kommunikationsstrategie für den Ernstfall.

In deutschen Krankenhäusern werden ca. 18 Millionen stationäre Patienten pro Jahr behandelt, von denen ca. 54.000 während ihres Aufenthalts reanimationspflichtig werden [1][2]. Bei diesen innerklinischen Reanimationen ist neben der strukturierten Behandlung entsprechend den aktuellen Leitlinien auch eine gute Teamarbeit und Kommunikation essenziell [2].

80 bis 90 Prozent der Behandlungsfehler in Notfallsituationen lassen sich auf menschliche Faktoren zurückführen [3]. Ein Drittel dieser Fehler sind durch mangelhafte Kommunikation bedingt [4]. Besonders die Reanimation in der Klinik ist durch multifaktorielle Spezifika der Akutmedizin prädestiniert für Kommunikationsfehler im Team [5].
Wieso neigen wir dazu, besonders bei der Reanimation nicht mehr auf die Kommunikation mit unseren Teammitgliedern zu achten?

Bei der innerklinischen Reanimation treffen zunächst verschiedene Teammitglieder zusammen. Die Schwierigkeit dabei ist, dass die Teams oft ad hoc entstehen und mehrere interdisziplinäre Berufsgruppen mit unterschiedlichen Kenntnisständen aufeinandertreffen. In den seltensten Fällen haben diese Teammitglieder in dieser Konstellation schon einmal zusammengearbeitet, was die Kommunikation umso mehr erschwert [5]. Zusätzlich ist eine Akutsituation wie die Reanimation für die Mitglieder des Teams auch immer eine Stresssituation. Stressoren haben für die einzelnen Teammitglieder wie auch für das 
gesamte Team negative Auswirkungen. Bei Stress kann es im Team zum Beispiel zur „Verantwortungsdiffusion“ kommen. Dieses Phänomen beschreibt, dass trotz genügender personeller Ressourcen offensichtliche Aufgaben nicht ausgeführt werden. Auch wird durch Stress die Koordination der Teammitglieder negativ beeinflusst und Lösungsversuche werden frühzeitig abgebrochen.

\section{"Closed loop“-Kommunikation}

Für eine effektive Kommunikation im Team gilt: „Gemeint ist nicht gesagt. Gesagt ist nicht gehört. Gehört ist nicht verstanden. Verstanden ist nicht getan. “ [6] Eine Kommunikationsstrategie, die diese Defizite verringern soll, ist die „Closed loop“- Kommunikation. Diese aus dem US-Militär stammende Form der Kommunikation beschreibt eine geschlossene Schleife der Gesprächsführung. Diese impliziert, dass auf jede Nachricht des Senders eine Antwort des Empfängers mit der Wiederholung der Nachricht folgt [7].

Ein Beispiel für eine „Closed loop“-Kommunikation in einer Reanimationssituation ließe sich wie folgt beschreiben: Person A sagt: „Person B, geben Sie jetzt $1 \mathrm{mg}$ Adrenalin i. v. " Dabei ist es wichtig, dass die Person, die die Anweisung ausführen soll, direkt mit Namen angesprochen wird und der Sender der Nachricht mit Körper-Augenkontakt die Botschaft unterstreicht. Die Botschaft sollte klar und knapp formuliert und unnötige Gespräche und Kommentare sollten vermieden werden [6]. Die Antwort auf die Ansprache sollte aus der Aufgabe sowie der Ausführung bestehen: Person B sagt: „Ja, verstanden. 1 mg Adrenalin i. v. Mache ich sofort. $1 \mathrm{mg}$ Adrenalin ist injiziert. " Mit dieser Kommunikationsstrategie sollen Missverständnisse verhindert und Fehler frühzeitig erkannt werden [8][9]. Hätte Person B nicht $1 \mathrm{mg}$ Adrenalin verstanden, sondern $3 \mathrm{mg}$, wäre es in der Wiederholung der Nachricht aufgefallen und hätte durch den Sender korrigiert werden können.

\section{Interaktion im Team}

In Reanimationssituationen sind das schnelle Erkennen und die richtige Therapie von zentraler Relevanz. Dabei kommt es nicht auf die Leistung der einzelnen Person an, sondern auf die Zusammenarbeit im interdisziplinären Team, da dies die Überlebenswahrscheinlichkeit des Patienten beeinflusst [10]. Besonders wichtig sind dabei die koordinierte Durchführung, ein effizienter Informationsaustausch sowie eine schnelle Rollenfindung im Team. Ein wichtiger Faktor ist die Übernahme der Teamleitung durch eines der Teammitglieder. Dieser Teamleitung kommt die Rolle zuteil, Aufgaben klar zu verteilen, Anweisungen an sein Team zu geben und die Überwachung der vorgegebenen Algorithmen der Reanimation zu gewährleisten. Dabei sollte er selbst nicht aktiv an den Reanimationsmaßnahmen teilnehmen, um den Überblick über die Situation zu behalten [11]. Neben der Teamführung ist auch die Teamarbeit des restlichen Teams unabdingbar. Hierbei ist es wichtig, dass Teammitglieder konstruktiv mitdenken und engagiert handeln. Sie sollten Bedenken sowie alternative Ideen einbringen, ohne die Autorität des Teamleiters zu untergraben. Damit sind sie eine wichtige Stütze für den Teamleiter und fördern eine gelungene Teamarbeit [12]. Dabei bestimmt vor allem die Beziehung zwischen den einzelnen Teammitgliedern und dem Teamleiter die Bereitschaft und das Engagement der Mitarbeit.

\section{Autoritätsgradient}

Der Autoritätsgradient beschreibt den hierarchischen Unterschied zwischen zwei Personen und dessen Auswirkungen [13]. Der Begriff wurde 1987 von Hawkins als humanfaktorieller Einfluss in das Vokabular der Flugsicherheit eingeführt, um entweder einen großen oder einen nicht existierenden hierarchischen Unterschied zwischen dem Flugkapitän und seiner Crew bezogen auf die Flugsicherheit aufzuzeigen [14]. Ein steile hierarchische Struktur wird hervorgerufen, wenn zwischen beiden Personen eine unterschiedliche Erfahrung im Beruf sowie ein großer Altersunterschied gegeben ist. Ein flaches Autoritätsgefälle entsteht, wenn beide Personen über vergleichbare Fähigkeiten verfügen oder miteinander befreundet sind [15]. Auch die Medizin ist sehr hierarchisch aufgebaut [4]. Deshalb wurde 1999 von dem Institute of Medicine ein Bericht veröffentlicht, der angelehnt an der Flugsicherheit deutlich machte, welche Auswirkungen der Autoritätsgradient in der Medizin hat. Daraus geht hervor, dass Ärzte und Pflegende, die in der hierarchischen Struktur nach ihrer eigenen Ansicht auf einem niedrigen Niveau stehen, oft aufgrund der Angst vor Sanktionen ihre Bedenken und Zweifel nicht äußern. Auch kann der Autoritätsgradient dazu führen, dass dem Teamleiter höhere Kompetenzen zugeschrieben werden, da man im Vorfeld positive Erfahrungen mit diesem gemacht hat und deshalb keine Notwendigkeit darin sieht, dessen Handeln infrage zu stellen [16]. Daher kann der Autoritätsgradient zu einer höheren Patientengefährdung führen [13].

\section{Ergebnisse bezogen auf die Art der Kommunikation}

Verschiedene Studien haben gezeigt, dass die Art der Kommunikation einen entscheidenden Einfluss auf ihre Effektivität hat. So haben El-Shafy et al. 2018 eine Studie in einem pädiatrischen Krankenhaus durchgeführt, die 89 Kindernotfälle (Schlaganfälle, Atemwegsverlegungen, Reanimationen) hinsichtlich der Kommunikation untersuchte. Methodisch wurden alle Vorfälle auf Video aufgezeichnet und von zwei unabhängigen Prüfern retrospektiv analysiert. Konkret wurde die zeitliche Differenz zwischen einer Anordnung und dessen Ausführung untersucht. Dabei wurde die Kommunikationsstrategie der „Closed loop“-Methode mit den herkömmlichen Kommunikationsformen verglichen und analysiert [17]. Insgesamt wurden 387 verbale Anordnungen aufgezeichnet. Jede Anordnung benötigte 


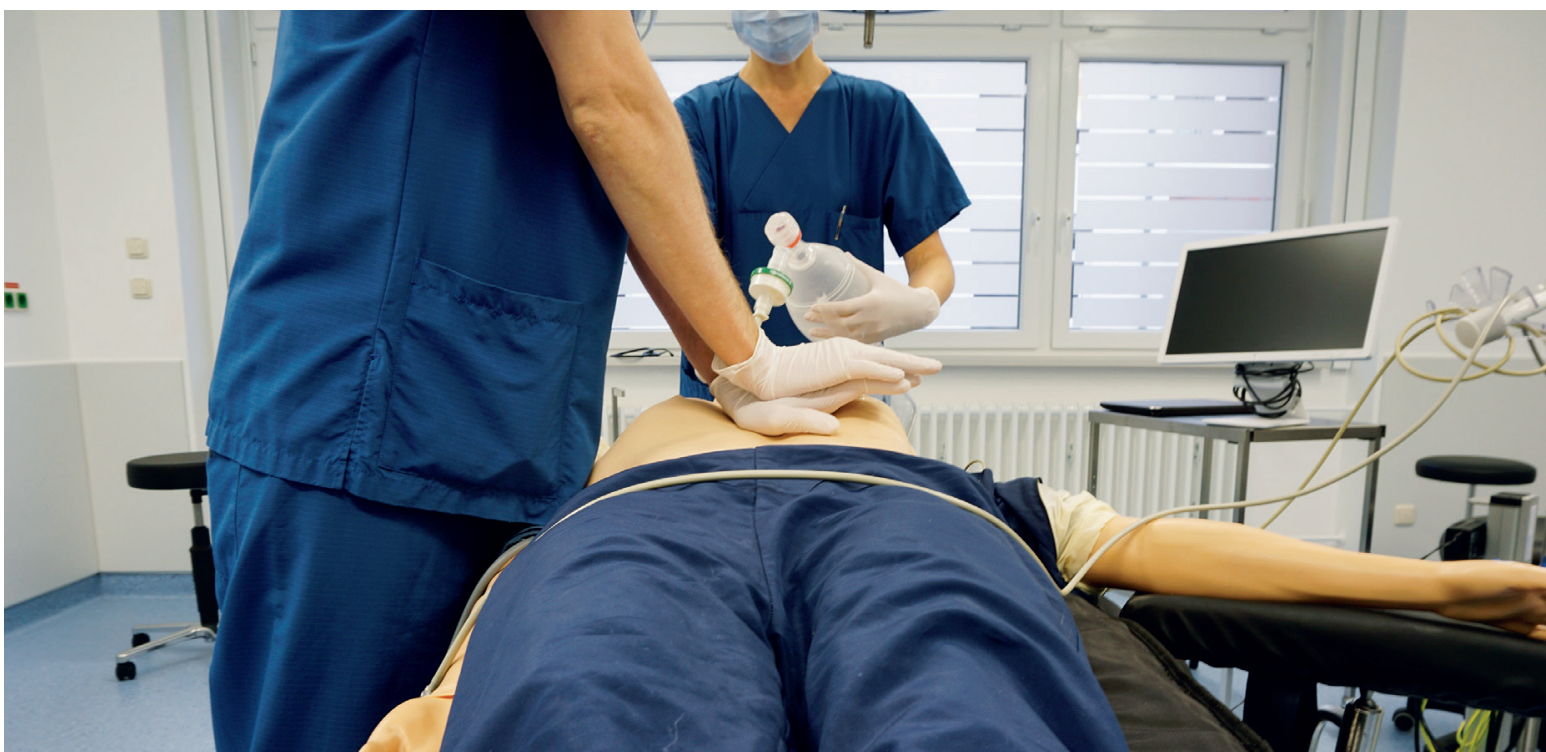

Teams, die regelmäßig an Simulationstrainings teilnehmen, verursachen signifikant weniger Fehler als Teams ohne Training.

im Durchschnitt 3,85 Minuten bis zu ihrer Erledigung. Es konnte gezeigt werden, dass die Zeit von der Anordnung bis zur Ausführung durch die „Closed loop“-Kommunikation signifikant reduziert werden konnte. Anordnungen mit der „Closed loop“-Kommunikation wurden 3,6-mal früher abgeschlossen als Anordnungen ohne die „Closed loop“-Kommunikation. Besonders sichtbar war der Effekt bei der Anforderung von Medikamenten. Mit der „Closed loop“-Kommunikation hat die Anordnung bis zur Ausführung eine Minute gedauert, ohne „Closed loop“-Kommunikation waren es durchschnittlich 4,5 Minuten. Die Ergebnisse haben auch gezeigt, dass ein Teamleiter die Teamarbeit und damit die Reanimation ebenfalls verbessert. Reanimationen, in denen der Teamleiter selbst auch Aufgaben übernommen hat, haben zu signifikant schlechteren Reanimationsergebnissen geführt [10]. Besonders innerklinisch werden die Reanimationsteams oft ad hoc zusammengestellt. Es gibt jedoch keine geltenden Richtlinien, welcher Mitarbeiter die Position des Teamleiters übernimmt. In der Literatur wird oft der anwesende Anästhesist als Beispiel für den Teamleiter genannt [16]. Gilligan et al. haben 2005 gezeigt, dass versiertes Fachpflegepersonal ebenfalls die Teamleitung übernehmen kann und dies keine explizite ärztliche Tätigkeit ist. Vielmehr haben die persönliche Erfahrung und die Überzeugung der eigenen Fähigkeiten, die Kompetenz, die Leitung des Teams zu übernehmen, einen höheren Stellenwert als die Berufsbezeichnung [18].

Studien haben zudem gezeigt, dass nicht nur die Reanimationsmaßnahmen trainiert werden sollten, sondern auch die Teamführung. Eine randomisierte kontrollierte Simulationsstudie mit 237 Medizinstudenten hat ergeben, dass die Reanimationen der Gruppen, die eine Teamleitungsinstruktion erhielten, signifikant besser verlaufen sind als die Reanimationen der Gruppen ohne Teamleitungsinstrukti- on. Der Langzeiteffekt ließ sich vier Monate nach der Instruktion immer noch nachweisen [19]. Sinnvoll könnte es daher sein, die jährlichen Reanimationsfortbildungen mit einer Teamführungsinstruktion zu ergänzen, um die Vorteile eines effektiven Teamleiters bei der Reanimation zu erhalten und damit die Reanimation zu verbessern. Wenn nicht genügend personelle Ressourcen zur Verfügung stehen, ist es schwierig, die Rolle des Teamleiters zu etablieren. Findet beispielsweise eine innerklinische Reanimation während des Nachtdienstes statt, ist es nicht möglich, noch eine zusätzliche Person als Teamleiter zu benennen, da alle Ressourcen für die Reanimation an sich benötigt werden. Trotzdem ist es ratsam, bei genügend Personal einen Teamleiter zu ernennen, um den zuvor beschriebenen Benefit bei der Reanimation nutzen zu können. Ebenfalls ratsam ist es, nach einer Reanimation im Team eine Nachbesprechung (Debriefing) durchzuführen. Die Intention des Debriefings ist es, Fehlerquellen zu identifizieren und aus diesen Fehlern lernen zu können [10].

\section{Resultate in Bezug auf das Team}

Inwieweit die Teamkommunikation eine Rolle spielt, hat die Simulationsstudie von St. Pierre et al. 2012 untersucht. Dabei wurde der Einfluss des Autoritätsgradienten analysiert. Bei dem Szenario wurde dem Oberarzt eine Regieanweisung ausgehändigt, die eine Narkoseeinleitung bei einem Patienten mit einem Ileus beinhaltete. Dabei wurden sieben Fehler in dieses Szenario eingebaut, die der Oberarzt bewusst nicht wahrnehmen oder selbst verursachen sollte. Die Intention der Autoren war, die „Bereitschaft von Assistenzärzten und Pflegekräften herauszufordern, sich um der Patientensicherheit willen mit Bedenken und Einwänden auch bei hierarchisch höher stehenden Personen zu Wort zu melden“ [16]. Die Fehler wurden nach verschiedenen Kriterien unterteilt: 
- lösbar, ohne zu kommunizieren,

- nur verbal lösbar,

- potenziell letale Handlung.

Die Ergebnisse zeigten, dass in den ersten vier Problemsituationen, die stillschweigend gelöst werden konnten, diese auch in 65 Prozent der Fälle so umgesetzt wurden. In patientengefährdenden Situationen haben 66 Prozent der Teilnehmer die Situation erkannt, aber nur 28 Prozent haben ihre Bedenken auch geäußert. Von den Äußerungen waren dann jeweils elf Prozent so eindeutig, dass der Fehler korrigiert werden konnte.

Ein Fehler bei dem fiktiven Szenario bestand darin, bei einem Blutdruck von $70 / 35$ mmHg ein weiteres blutdrucksenkendes Medikament zu verabreichen. Für alle Beteiligten des Szenarios sollte klar sein, dass diese Medikation absolut kontraindiziert ist und letale Folgen haben kann. Wieso wurde also trotzdem nicht gehandelt? Als mögliche Einflussgröße wird in der Studie ganz eindeutig der Autoritätsgradient angegeben. Dieser hindert den freien Informationsfluss zwischen Sender und Empfänger. Besonders hinderlich sind dabei die eigene Rollendefinition und die vermeintliche Stellung in der Hierarchie. So hat ein neuer Assistenzarzt beispielsweise die Befürchtung, dass seine Anmerkung, dass dieses Medikament nicht indiziert ist, zufolge haben könnte, dass er als besserwisserisch gesehen wird bzw. seine Kompetenz überschreitet und dies dann negative Konsequenzen für ihn hat. Demgegenüber haben Führungskräfte in der Medizin die Sorge vor öffentlicher Bloßstellung und der Infragestellung ihrer Kompetenz. „Klinische Kompetenz und Expertise sind das Resultat eines über Jahrzehnte dauernden Lernprozesses, der in einer ganzheitlichen Wahrnehmung komplexer Situationen und in einer mühelosen Anwendung intuitiver und analytischer Lösungsstrategien mündet. " [16]

Daher ist die Sorge unbegründet, dass die Kompetenz aufgrund produktiver, sicherheitsrelevanter Anmerkungen infrage gestellt wird. Abgesehen davon sollten die Priorität und der Fokus auf dem Wohlergehen des Patienten liegen und nicht auf dem persönlichen Ansehen. Der Autoritätsgradient ist nicht nur klinisch bedeutsam, wenn die Autorität der Führungsperson negativ besetzt ist, sondern auch, wenn die Führungsperson durch ihre Persönlichkeit und fachliche Expertise geachtet wird. Das hatte bei der Simulationsstudie zur Folge, dass 23 Prozent der Teilnehmer das eigene pathophysiologische Verständnis hinterfragen, aber nicht das des geachteten Oberarztes. Bei zwölf Prozent wurde die Anordnung überhaupt nicht hinterfragt, da „blindes Vertrauen“ beschrieben wurde und die Verantwortung demnach vollständig beim Oberarzt gelegen hat. Das Paradoxe dabei ist, dass die Oberärzte, die in der Studie als geachtet beschrieben werden, in den meisten Fällen für konstruktive Kritik oder Unterstützung offen sind. Diese werden aber nicht geäußert: „Weil man nur gute Erfahrungen mit der Kompetenz der Führungsperson gemacht hat, sieht man keine Notwendigkeit, deren Handlungen kritisch zu begleiten oder einen wahrgenommenen Informationskonflikt anzusprechen. “[16]

Die Medizin wird häufig mit der Luftfahrt verglichen, da beide Disziplinen in einem Hochrisikobereich mit einem hohen Komplexitätsgrad interagieren [6][20]. In der Luftfahrt ist man jedoch davon abgekommen, eine steile Hierarchie zu fördern. Durch sogenannte „Team Skills“ werden die Kommunikation an sich sowie verschiedene Kommunikationsmethoden trainiert und etabliert [15]. Der Aussage, dass unerfahrene Teammitglieder die Anordnung erfahrener Mitglieder nicht infrage stellen sollten, stimmten 25 Prozent der anästhesiologischen und 40 Prozent der chirurgischen Oberärzte zu. Im Vergleich dazu waren nur zwei Prozent der Piloten mit dieser Aussage einverstanden [20]. Diese Differenz der prozentualen Anteile verdeutlicht die Weiterentwicklung und Abflachung der Hierarchie in der Luftfahrt und das Potenzial, die unglücklicherweise noch zu steilen Hierarchien in der Medizin zu verringern.

\section{Simulationstraining}

Um die Effektivität der Reanimation oder anderer Notfallsituationen zu verbessern, hat sich das Simulationstraining etabliert. Die Ergebnisse legen dar, dass Teams, die regelmäßig an Simulationstrainings teilnehmen, signifikant weniger Fehler verursachen und schneller interagieren als Teams ohne Training [21][22]. Trotz dieser bekannten Ergebnisse werden Simulationstrainings in Deutschland spärlich eingesetzt. Generell ist es in Deutschland im Gegensatz zum angloamerikanischen Raum nicht verpflichtend, als medizinisches Personal an Reanimationsfortbildungen teilzunehmen. Jedes Krankenhaus kann selbst darüber bestimmen, ob und im welchem Intervall Reanimationsfortbildungen angeboten werden [23]. Um von den positiven Effekten des Trainings zu profitieren, empfiehlt es sich, eine jährliche Teilnahme des medizinischen Personals (Pflegende und Ärzte) für das Reanimationstraining verpflichtend einzuführen. Optimal wäre es, wenn das Personal, das am häufigsten mit Reanimationssituationen in Berührung kommt, wie die Fachbereiche der Anästhesie, Intensiv- sowie Notfallmedizin, dieses im Rahmen eines Simulationstrainings mit Videodebriefing durchführen könnten.

\section{Zusammenfassung}

Die Relevanz der Kommunikation bei der innerklinischen Reanimation ist in Deutschland konstituiert. Die vorliegenden Ergebnisse zeigen, dass die Kommunikation bei einer innerklinischen Reanimation eine Auswirkung auf das Outcome des Patienten hat. Am Beispiel der „Closed loop“-Kommunikation konnte veranschaulicht werden, dass die Zeit zwischen der Anordnung und der Ausführung dieser Anordnung signifikant reduziert werden konnte und die Fehlerrate verringert wurde. Des Weiteren hat die Be- 
deutung des Teamleiters ebenfalls positive Auswirkungen auf die Zeitersparnis bei der Reanimation, ungeachtet dessen, ob eine Pflegekraft oder ein Arzt die Rolle einnimmt. Es konnte dargelegt werden, dass sowohl die Basismaßnahmen bei der Reanimation als auch die Kommunikation im Team trainiert werden sollten. Daher ist die Etablierung eines Kommunikationstrainings in den Reanimationsfortbildungen empfehlenswert, wie es auch die US-amerikanischen Leitlinien empfehlen. Zusätzlich konnte festgestellt werden, dass steile hierarchische Strukturen die Kommunikation behindern und einen Austausch von Fehlern im Hinblick auf die Patientensicherheit negativ beeinflussen. Hingegen ist der nachfolgenden Reflexion der Reanimation im Team, dem sogenannten „Debriefing“, eine positive Verbesserung der Reanimationsqualität nachzuweisen. Zusätzlich sollte jährlich das Reanimationstraining verpflichtend für das medizinische Personal sein.

Prospektiv sollte aufgrund der hohen Relevanz von innerklinischen Reanimationen weitere Forschung betrieben und die Bedeutung der Kommunikation zu diesem Thema mehr in den Forschungsfokus gerückt werden.

\section{Autorinnen/Autoren}

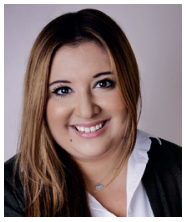

Anna Henn, B. A.

Medizinpädagogin, Praxisanleiterin, Gesundheits- und Krankenpflegerin Fachbereich Anästhesie, Kliniken Maria Hilf Mönchengladbach. Zurzeit Teilnehmerin der

Fachweiterbildung Anästhesie und Intensivpflege.

E-Mail: anna.henn@mariahilf.de

\section{Literatur}

[1] Deutsche Gesellschaft für Anästhesiologie und Intensivmedizin. Deutsches Reanimationsregister. Online unter bit. ly/2ZuVs9h, letzter Zugriff 27.12.2019

[2] Burghofer K, Ruchholz S, Seekamp A et al. Kommunikation am Notfallort. Notfallmedizin up2date 2008, 3(2): 121-32

[3] Happel O, Papenfuß T, Kranke P. Schockraummanagement - Simulation, Teamtraining und Kommunikation für eine bessere Traumaversorgung. Anästhesiol Intensivmed Notfallmed Schmerzther 2010; 45(6): 408-15 - doppelt, Verweise anpassen

[4] Tewes R. Wie bitte? Kommunikation in Gesundheitsberufen. Heidelberg: Springer; 2016

[5] Burghofer K, Lackner C. Kommunikation. Risikofaktor in der Akutmedizin. Notfall- und Rettungsmedizin. Heidelberg: Springer; 2012

[6] Happel O, Papenfuß T, Kranke P. Schockraummanagement - Simulation, Teamtraining und Kommunikation für eine bessere Traumaversorgung. Anästhesiol Intensivmed Notfallmed Schmerzther 2010; 45(6): 408-15

[7] American Heart Association. Best Practices in Hospital Resuscitation Response Teams. American Heart Association.
6. Mai 2015. Online unter bit.ly/2s84yMR, letzter Zugriff 09.01.2020

8] Major Trauma Guidelines \& Education. Victorian State Trauma System. Effetive Communication. Online unter bit.ly/2Sw10Pb, letzter Zugriff 27.12.2019

[9] Davis W, Jones S, Crowell-Kuhnberg AM et al. Operative team communication during simulated emergencies: Too busy to respond? Surgery 2017, 161(5): 1348-56

[10] Becker C, Marsch S, Eppich W et al. Führen optimierte Teamarbeit und Führungsverhalten zu besseren Reanimationsergebnissen? Was können wir daraus für andere Notfälle lernen? Notfall + Rettungsmedizin 2018, 3: 171-6

[11] Künzle B, Kolbe M, Grote G. Ensuring patient safety through effective leadership behaviour: a literature review. Safety Science 2010, 48(1): 1-17

[12] Gibbons A, Bryant D. Followership: the forgotten part of doctors' leadership. BMJ 2012, 345: e6715

[13] Stemmler J, Hecker U. Notfallkommando - Kommunikation in Notfallsituationen für Gesundheitsberufe. Heidelberg: Springer; 2016

[14] Hawkins F. Human Faktors in Flight. London (GB): Roudlege Verlag; 1987

[15] European Helicopter Safety Team. Schulung und Prüfungen für Notverfahren und anormale Verfahren in Hubschraubern. Online unter bit.ly/2PZUgHG, letzter Zugriff 27.12.2019

[16] St. Pierre M, Scholler, A, Strembski D et al. Äußern Assistenzärzte und Pflegekräfte sicherheitsrelevante Bedenken? Simulatorstudie zum Einfluss des „Autoritätsgradienten“. Anaesthesist 2012, 61: 857-66

[17] Abd El-Shafy IA, Delgado J, Akerman M et al. Closed-Loop Communication Improves Task Completion in Pediatric Trauma Resuscitation. J Surg Educ 2018, 75(1): 58-64

[18] Gilligan P, Bhatarcharjee C, Knight G et al. To lead or not to lead? Prospective controlled study of emergency nurses' provision of advanced life support team leadership. Emerg Med J 2005, 22(9): 628-32

[19] Hunziker S, Bühlmann C, Tschan F et al. Brief leadership instructions improve cardiopulmonary resuscitation in a high-fidelity simulation: a randomized controlled trial. Crit Care Med 2010, 38(4): 1086-91

[20] Simon R, Langford V, Locke A et al. A successful transfer of lessons learned in aviation psychology and flight safety to health care. The MedTeams System 2000, 45-9

[21] Falcone RA, Daugherty M, Schweer L et al. Multidisciplinary pediatric trauma team training using high-fidelity trauma simulation. J Pediatr Surg 2008, 43(6): 1065-71

[22] Schröder T, Rau J, Volk T. Simulation-based medical team training to improve patient safety in the operating room. Perioperative Medizin 2009, 1(1): 44-9

[23] Siebig S, Kues S, Klebl F et al. Herz-Kreislauf-Stillstand - Wer reanimiert und wie wird trainiert? Ergebnisse einer Umfrage in deutschsprachigen Krankenhäusern. Dtsch Arztebl 2009, 106(5): 65-70

Bibliografie

DOI https://doi.org/10.1055/a-1088-5117

intensiv 2020; 28: 68-72

(c) Georg Thieme Verlag KG Stuttgart · New York

ISSN 0942-6035 